\title{
Optimal control of particle separation in inertial microfluidics
}

\author{
Christopher Prohm ${ }^{1, a}$, Fredi Tröltzsch ${ }^{2}$, and Holger Stark ${ }^{1}$ \\ 1 Institute of Theoretical Physics, Technische Universität Berlin, Hardenbergstr. 36, 10623 Berlin, Germany \\ 2 Institut für Mathematik, Technische Universität Berlin, Straße des 17. Juni 136, 10623 Berlin, Germany
}

Received 12 August 2013 and Received in final form 23 September 2013

Published online: 25 October 2013

(c) The Author(s) 2013. This article is published with open access at Springerlink.com

\begin{abstract}
Recently, inertial mircofluidics has emerged as a promising tool to manipulate complex liquids with possible biomedical applications, for example, to particle separation. Indeed, in experiments different particle types were separated based on their sizes (A.J. Mach, D. Di Carlo, Biotechnol. Bioeng. 107, 302 (2010)). In this article we use a theoretical study to demonstrate how concepts from optimal control theory help to design optimized profiles of control forces that allow to steer particles to almost any position at the outlet of a microfluidic channel. We also show that one specific control force profile is sufficient to guide two types of particles to different locations at the channel outlet, where they can be separated from each other. The particles just differ by their size which determines the strength of the inertial lift forces they experience. Our approach greatly enhances the efficiency of particle separation in the inertial regime.
\end{abstract}

\section{Introduction}

Sorting particles is important for a wealth of technological applications ranging from biological and chemical to medical systems [1-3]. Especially, microfluidics and its labon-a-chip applications [4] are used to manipulate particles in a Poiseuille flow and to steer them to different lateral locations in a channel in order to separate different particle types from each other [1-3]. This procedure is also called continuous flow separation. At low Reynolds numbers, where fluid inertia can be neglected, spherical particles just follow the streamlines of fluid flow [5]. However, in biomedical applications often the Reynolds number is above one and the so-called lift forces drive particles to specific lateral positions in a microfluidic channel [6-11]. This inertial focussing is then used to separate bioparticles such as red blood cells and bacteria from each other or to filter out one particle type $[9,11-13]$. The purpose of this article is to demonstrate how methods inspired by optimal control theory [14-16] help to determine control forces, which are additionally applied to flowing particles and are able to redirect or steer them to specific locations at an channel outlet. As we demonstrate, this largely improves the capability of inertial microfluidics to separate particles.

At Reynolds numbers larger than one, particles migrate towards preferential positions in a channel depending on the geometry of the channel cross-section. As first observed by Segré and Silberberg in circular channels, the

\footnotetext{
a e-mail: christopher.prohm@tu-berlin.de
}

particles accumulate on an annulus halfway between the channel center and the wall [17]. In a square channel they settle to four positions located between the channel center and the centers of the channel faces [18]. To explain inertial focussing, already Segré and Silberberg introduced the inertial lift force [17]. It combines two contributions. The first one is induced by the shear gradients of the Poiseuille flow and drives particles to positions with higher shear gradients, while the wall-induced contribution pushes particles towards the channel center [19].

Channels with rectangular cross-sections allow to independently tune the magnitude of the lift forces in two directions by varying the aspect ratio. If this ratio is sufficiently large, lift forces along the longer cross-sectional axis point towards the center plane of the channel, as demonstrated in finite-element studies [18]. As a result the particle dynamics becomes two-dimensional. This has repeatedly been demonstrated in experiments [8,13, 20,21]. In the following, we will use this fact in our investigations.

Lift forces are caused by the non-linear term in the Navier-Stokes equations. Semi-analytic studies valid for small particle size compared to the channel diameter are based on the method of matched asymptotic expansion $[22,23]$. They reproduce experimental results in the range of intermediate Reynolds numbers. In microfluidic systems particle sizes are typically comparable to the channel diameter and one has to resort to fully numerical methods to determine the lift forces. Such studies include simulations using finite elements [18], lattice Boltzmann [24], and multi-particle collision dynamics [25]. 
Inertial lift forces show a pronounced scaling with the particle radius $[18,22]$. For example, they direct large particles to specific lateral positions in the channel. Smaller particles only experience small forces which take longer to establish focussed particle trains. Therefore, smaller particles keep their initially uniform distribution in the channel cross-section for longer times. Mach et al. have used this effect to separate bacteria from red blood cells which only works due to the sufficiently large difference in particle size [13]. Particle separation would be more effective, in particular for particles of similar size, if one could direct them to defined lateral positions within the channel. In the following we use methods from optimal control theory [14] to design the action of control forces that are able to steer particles to almost any cross-sectional position. We also demonstrate that our approach can separate two particle types of similar size using solely the difference in the lift-force profiles.

The theory of optimal control of systems that are describable by partial differential equations is widely employed in different fields such as fluid mechanics, microelectronics, crystal growth, vascular surgery, and cardiology [14]. Here we apply it to the Smoluchowski equation, which governs the microfluidic system, and determine external control forces for particle steering. Optimal control theory of stochastic systems is commonly used in finance where the inherent uncertainty of stock prices is modeled by a Langevin equation [26]. For example, Merton's famous portfolio problem deals with an investiment strategy and surprisingly permits an analytic solution [27]. The field of reinforcement learning, applied, for example, in machine learning in computer science, game theory, and genetic algorithms, develops strategies on how an agent can choose its actions to maximize expected future rewards [28]. Recently, optimal control has also been applied to stochastic thermodynamics [29].

Section 2 outlines how we use optimal control theory for steering and separating particles. We also explain our numerical method to solve the relevant equations. In sect. 3 we review necessary facts from inertial microfluidics and present profiles of lift forces to be used for the following investigations. Finally, in sect. 4 we demonstrate how optimal control theory helps to steer particles and to separate them based on their size. We finish with a summary and conclusions in sect. 5 .

\section{Optimal control of particle separation}

In the following we outline the theory to perform optimal control in order to steer particles in a microchannel to specific lateral positions. As illustrated in fig. 1, a single particle with position $\vec{x}=(x, z)$ moves in a rectangular domain $-w \leq x \leq w, 0 \leq z \leq L$ under the influence of a pressure-driven Poiseuille flow that causes a drift velocity $v_{z}(x)$ along the axial $z$-direction which is fixed in the following. An inertial lift force $f_{\text {lift }}(x)$ acts along the lateral $x$-direction which we explain in sect. 3.2 . In addition, we introduce a control force $u(\vec{x})$ to steer particles. We will comment on its experimental realization in sect. 3.3.

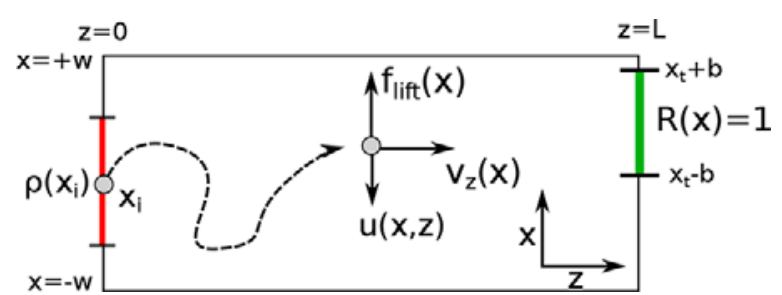

Fig. 1. The schematic control setup. We try to steer a single particle, whose initial position is distributed according to $\rho_{0}\left(x_{i}\right)$ (red bar), towards a given target interval at the channel end (green bar). The target is specified by the rewards function $R(x)$, which is one inside the target interval and zero outside. The particle experiences lift $\left[f_{\text {lift }}(x)\right]$ and control $[u(x, z)]$ forces along the lateral $x$-direction and drifts with velocity $v_{z}(x)$ in the $z$-direction.

Since the particle also performs thermal Brownian motion, we set up the Smoluchowski equation for the probability density $\rho(\vec{x}, t)$ to find the particle at position $\vec{x}$ at time $t$,

$$
\frac{\partial}{\partial t} \rho(\vec{x}, t)=-\vec{\nabla} \cdot \vec{j}=\mathcal{L} \rho(\vec{x}, t),
$$

where the differential operator $\mathcal{L}$ is specified below. The probability current $\vec{j}$ has contributions from deterministic drift motion and thermal diffusion. Its respective components along the lateral and axial directions are given by

$$
\begin{aligned}
& j_{x}(\vec{x})=\left[\xi^{-1}\left[f_{\text {lift }}(x)+u(\vec{x})\right]-D \frac{\partial}{\partial x}\right] \rho(\vec{x}, t), \\
& j_{z}(\vec{x})=v_{z}(x) \rho(\vec{x}, t),
\end{aligned}
$$

where $\xi$ is the Stokes friction coefficient and $D=k_{B} T / \xi$ the diffusion constant of the particle. Along the axial direction we neglect thermal motion. With these currents the operator $\mathcal{L}$ assumes the form

$$
\mathcal{L}[u]=-\frac{\partial}{\partial x}\left[\xi^{-1}\left[f_{\mathrm{lift}}(x)+u(\vec{x})\right]-D \frac{\partial}{\partial x}\right]-\frac{\partial}{\partial z} v_{z}(\vec{x}) .
$$

As the particles cannot leave the microchannel through the bounding walls, the probability current in the $x$-direction has to satisfy $j_{x}=0$ at the lateral boundaries $x= \pm w$.

\subsection{Steering one particle}

We consider a single particle which we try to steer from the channel inlet $(z=0)$ into a given target interval $\left[x_{t}-b, x_{t}+b\right]$ at the channel outlet $(z=L)$ by applying an appropriate control force $u(\vec{x})$ (see fig. 1). Initially, the probability to find the particle inside the channel is zero, while its initial position $x_{i}$ at the inlet is distributed according to $\rho_{0}\left(x_{i}\right)$. The probability $J$ of the particle to exit through the target is given by integrating the probability current over the target interval,

$$
J=\int_{0}^{\infty} \mathrm{d} t \int_{-w}^{+w} \mathrm{~d} x j_{z}(x, z=L, t) R(x),
$$


where we introduced the rewards function $R(x)$ from optimal control theory [28] which in our case becomes $R(x)=1$ for $x \in\left[x_{t}-b, x_{t}+b\right]$ and zero otherwise. We also integrate over all times, as we are only interested whether the particle reaches its target, and not how long this takes.

Determining an optimal control force $u$ by maximizing the probability $J$ is not a well-defined mathematical problem [14]. First, it allows for arbitrarily large control forces and second, so far the control foces can vary on arbitrarily small length scales. To remedy both problems, we add two regularization terms to $J$. First, to avoid excessively high control forces, we introduce the quadratic control cost $\frac{1}{2} \lambda^{2} \int_{\Omega} \mathrm{d}^{2} x|u(\vec{x})|^{2}$, where $\Omega$ is the spatial domain of the channel. Second, to obtain smooth profiles for the control force, we use $\frac{1}{2} \int_{\Omega} \mathrm{d}^{2} x\left[\left(\lambda_{x} \frac{\partial}{\partial x} u(\vec{x})\right)^{2}+\left(\lambda_{z} \frac{\partial}{\partial z} u(\vec{x})\right)^{2}\right]$ that penalizes large gradients in $u$. Now, instead of the probability $J$, we maximize what optimal control theory calls the objective functional

$$
\begin{aligned}
J^{\text {tot }}= & \int_{0}^{\infty} \mathrm{d} t \int_{-w}^{+w} \mathrm{~d} x_{f} j_{z}\left(x_{f}, L, t\right) R\left(x_{f}\right) \\
& -\frac{1}{2} \int_{\Omega} \mathrm{d}^{2} x\left[\lambda^{2}[u(\vec{x})]^{2}+\lambda_{x}^{2}\left(\frac{\partial}{\partial x} u(\vec{x})\right)^{2}\right. \\
& \left.+\lambda_{z}^{2}\left(\frac{\partial}{\partial z} u(\vec{x})\right)^{2}\right] .
\end{aligned}
$$

A necessary condition for a maximum of $J^{\text {tot }}$ is that its variation vanishes, $\delta J^{\text {tot }}[u]=0$. However, the particle probability density has to obey the Smoluchowski equation (1) and one includes this constraint for the variation with a Lagrange multiplier [14]. As demonstrated in appendix A, the control force $u(\vec{x})$ maximizing the objective function then satisfies gradient equation

$$
u(\vec{x})-\frac{\lambda_{x}^{2}}{\lambda^{2}} \frac{\partial^{2}}{\partial x^{2}} u(\vec{x})-\frac{\lambda_{z}^{2}}{\lambda^{2}} \frac{\partial^{2}}{\partial z^{2}} u(\vec{x})=u_{0}(\vec{x}),
$$

where $u_{0}(\vec{x})$ denotes the part of the optimal control force $u$ that is not related to the regularization terms,

$$
u_{0}(\vec{x})=\frac{\xi^{-1}}{\lambda^{2}} W(\vec{x}) \frac{\partial}{\partial x} V(\vec{x}) .
$$

Here we introduced the time-integrated or cumulated density $W(\vec{x})=\int_{0}^{\infty} \mathrm{d} t \rho(\vec{x}, t)$ that satisfies the stationary Smoluchowski equation

$$
\mathcal{L}[u] W(\vec{x})=0,
$$

with the boundary condition of vanishing current at the channel walls $\left(j_{x}=0\right)$ and $W(x, z=0)=\rho_{0}(x)$ at the inlet. We can also call $W(\vec{x})$ the state function, which satisfies the state equation (9). Introducing the adjoint operator

$$
\mathcal{L}^{+}[u]=\xi^{-1}\left[f_{\text {lift }}(x)+u(x, z)\right] \frac{\partial}{\partial x}+v_{z}(x) \frac{\partial}{\partial z}+D \frac{\partial^{2}}{\partial x^{2}},
$$

the adjoint state function $V(\vec{x})$ satisfies the stationary Kolmogorov backward or adjoint equation

$$
\mathcal{L}^{+}[u] V(\vec{x})=0,
$$

with the respective boundary conditions $\frac{\partial}{\partial x} V(\vec{x})=0$ at the channel wall and $V\left(x_{f}, z=L\right)=R\left(x_{f}\right)$ at the channel outlet. Since the Kolmogorov backward equation integrates backward in time, $V(\vec{x})$ gives the probability that a particle starting at $\vec{x}$ reaches the target with certainty. Therefore, $V$ is the so-called value function.

Note that all three partial differential equations (7), (9), and (11) are coupled to each other and have to be solved self-consistently. The resulting optimized control force is smooth on length scales $\lambda_{x} / \lambda, \lambda_{z} / \lambda$. Since $u(\vec{x}) \propto \partial V(\vec{x}) / \partial x$ according to eqs. (7) and (8), the optimal control force pushes the particle along the gradient of the value function and thereby increases the probability that the particle reaches the target at the outlet. We discuss this point in more detail in sect. 4.1.

We add a last remark. In general, the control force $u(\vec{x})$ maximizing the objective functional will be larger than some maximal value $u_{\max }$ available in a concrete setting. To obey the bounds $|u(x)|<u_{\max }$, we determine the optimal control of the unconstrained problem and project it on the admissible set defined by the bounds

$$
u(\vec{x}) \rightarrow\left\{\begin{aligned}
u(\vec{x}) & \text { if }|u(\vec{x})|<u_{\max }, \\
u_{\max } & \text { if } u(\vec{x}) \geq u_{\max } \\
-u_{\max } & \text { if } u(\vec{x}) \leq-u_{\max }
\end{aligned}\right.
$$

Though the result is not the exact optimal control, it gives a good approximation that works very well for the applications.

\subsection{Separation of particles}

The problem of separating particles of different types $i$ is a direct extension of the single particle problem. We assume that the particles differ in their respective lift forces $f_{\text {lift }}^{(i)}$ as well as friction coeffcients $\xi_{i}$. However, the control force $u(\vec{x})$ acting on the particles is the same for all particles.

In sect. 4.2 we will look at two particle types and maximize the probability that the first and the second particle type reach their respective targets. In the simplest case of a dilute system, where interactions between particles are negligible, the probability for this event is the product of the individual probabilities,

$$
J=J_{1}[u] J_{2}[u] .
$$

Again, to obtain the total objective function $J^{\text {tot }}$, we add the two regularization terms for $u(\vec{x})$. We perform the variation of $J^{\text {tot }}$ as before but have to apply the product rule to $J=J_{1} J_{2}$. Then the optimal control force without the regularization terms becomes

$$
u_{0}(\vec{x})=\frac{1}{\lambda^{2}} \sum_{i=1}^{2} \frac{J}{J_{i} \xi_{i}} W_{i}(\vec{x}) \frac{\partial}{\partial x} V_{i}(\vec{x}) .
$$

Here the contribution for each particle type is weighted by the probability $J / J_{i}$ that particles of the other type reach their respective targets. $W_{i}$ and $V_{i}$ are the respective timeintegrated probability density and the value function for particle type $i$. 


\subsection{Numerical evaluation}

We solve eqs. (7), (9), and (11) numerically by discretizing them on a rectangular grid equal to the $x, z$ lattice. It has mesh sizes $\Delta x$ and $\Delta z$ and the coordinate basis $\hat{e}_{x}, \hat{e}_{z}$. However, if one directly discretizes the operator $\mathcal{L}$ using finite differences, one obtains a highly unstable scheme which is basically due to the fact that the probability density can become negative [30]. To remedy this shortcoming, we introduce on the grid a random walk process with drift which is equivalent to our Smoluchowski equation (1) with (4). This procedure is also known as Markov chain approximation [30]. While it has originally been designed for stochastic processes, it has also been applied to deterministic systems [31].

We denote the probability to jump to lattice position $\vec{x}_{i}$ from position $\vec{x}_{j}$ by $P_{i j}(u)$, where we only allow jumps to nearest neighbors and the jump vector is $\Delta \vec{x}_{i j}=\vec{x}_{i}-\vec{x}_{j}$. The lattice process and the Smoluchowski equation are equivalent when they both give the same mean displacement and mean square displacement for the jump time $\Delta t$

$$
\begin{aligned}
\sum_{i \in \mathrm{NN}(j)} P_{i j}(u) \Delta \vec{x}_{i j}= & \xi^{-1}\left[f_{\text {lift }}\left(\vec{x}_{j}\right)+u\left(\vec{x}_{j}\right)\right] \hat{e}_{x} \Delta t \\
& +v_{z}\left(\vec{x}_{j}\right) \hat{e}_{z} \Delta t \\
\sum_{i \in \mathrm{NN}(j)} P_{i j}(u) \Delta \vec{x}_{i j} \otimes \Delta \vec{x}_{i j}= & 2 D \hat{e}_{x} \otimes \hat{e}_{x} \Delta t \\
& +\mathcal{O}\left(\Delta t^{3 / 2}\right),
\end{aligned}
$$

where $\mathrm{NN}(j)$ indicates the four nearest neighbors of position $\vec{x}_{j}$. To satisfy eqs. (15) and (16), we choose the transition probabilities as

$$
P_{i j}(u)=\left\{\begin{array}{c}
\xi^{-1}\left(\left[f_{\text {lift }}(\vec{x})\right]^{+}+[u(\vec{x})]^{+}\right) \frac{\Delta t}{\Delta x}+\frac{D \Delta t}{(\Delta x)^{2}} \\
\quad \text { if } \Delta \vec{x}_{i j}=+\Delta x \hat{e}_{x}, \\
\xi^{-1}\left(\left[f_{\text {lift }}(\vec{x})\right]^{-}+[u(\vec{x})]^{-}\right) \frac{\Delta t}{\Delta x}+\frac{D \Delta t}{(\Delta x)^{2}} \\
\quad \text { if } \Delta \vec{x}_{i j}=-\Delta x \hat{e}_{x}, \\
v_{z} \frac{\Delta t}{\Delta z} \quad \text { if } \Delta \vec{x}_{i j}=+\Delta z \hat{e}_{z}, \\
0 \quad \text { if } \Delta \vec{x}_{i j}=-\Delta z \hat{e}_{z}, \\
\xi^{-1}\left(u_{\max }-|u|\right) \frac{\Delta t}{\Delta x} \quad \text { if } \vec{x}_{i}=\vec{x}_{j} .
\end{array}\right.
$$

Here we defined $\left[f_{\text {lift }}(\vec{x})\right]^{ \pm}=\max \left( \pm f_{\text {lift }}(\vec{x}), 0\right)$ and similarly $[u(\vec{x})]^{ \pm}$. This means that positive forces contribute to jump probabilities in the positive $x$-direction, whereas negative forces are taken into account in jumps along the negative $x$-direction. We ensure that the jump probabilities sum to one $\left(P_{j j}+\sum_{i \in \mathrm{NN}(\mathrm{j})} P_{i j}=1\right)$ by choosing the jump time $\Delta t$ accordingly. As the drift forces depend on the grid position $\vec{x}_{j}$, the time step $\Delta t_{j}$ also becomes dependent on $\vec{x}_{j}$. Since we are not interested in the time behavior of our system, this does not cause any problems. In principle, $\Delta t_{j}$ would also depend on the control force $u\left(\vec{x}_{j}\right)$, which leads to numerical artifacts. To avoid this, we also include a probability $P_{j j}$ in eqs. (17) which does not contribute to the mean displacement and mean square displacement. However, when calculating $\Delta t_{j}$, the term proportional to $|u|$ just cancels the respective terms in $P_{i j}$. The maximal control force $u_{\max }$ is added to ensure $P_{j j}>0$. The non-zero $P_{j j}$ slows down the relaxation of the probability distribution $W_{i}$. Since we only determine the steady state, this is not important for us.

Finally, we also include the boundary conditions in the jump probabilities. To implement the zero probability current at the channel walls at $x= \pm w$, we set the probability for jumping across the wall to zero and add its contribution to the probability $P_{j j}$ of staying at the boundary. We include the boundaries at $z=0$ (inlet) and $z=L$ (outlet) by setting the jump probability to move forward along the $z$-direction equal to one and all other transition probabilities to zero. This means particles enter the system with certainty at the inlet and leave it once they have reached the outlet.

Now, the time dependence of the probability $W_{i}$ obeys

$$
W_{i}^{(n+1)}=\sum_{j} P_{i j}(u) W_{j}^{(n)}+\Delta t_{i} \rho_{i},
$$

which we iterate until we find the steady-state solution. The second term on the right-hand side ensures that the probability density at the inlet is always $\rho_{i}=\rho_{0}\left(x_{i}\right) \Delta x$.

Equation (18) shows that in our lattice approach the Smoluchowski operator $\mathcal{L}$ is replaced by

$$
L_{i j}=\frac{1}{\Delta t_{i}}\left(P_{i j}-\delta_{i j}\right) .
$$

Since the stationary Kolmogorov backward equation (11) is governed by the adjoint Smoluchowski operator $\mathcal{L}^{+}$, the dynamics of $V_{i}$ is governed by the transposed matrix $L^{+}$ with elements $\left[L^{+}\right]_{i j}=\left(P_{j i}-\delta_{i j}\right) / \Delta t_{i}$. So we determine the steady-state solution of

$$
V_{i}^{(n+1)}=\sum_{j} P_{j i}(u) V_{j}^{(n)}+\Delta t_{i} R_{i},
$$

where the reward function $R_{i}$ determines the boundary condition at the outlet at $z=L$.

To discretize eq. (7), we interpret it as a stationary diffusion equation with additional loss $(-u)$ and source $\left(u_{0}\right)$ terms. For diffusion along $x$ - and $z$-direction with the respective diffusion constants $\lambda_{x}^{2} / \lambda^{2}$ and $\lambda_{z}^{2} / \lambda^{2}$, we introduce jump probabilities $\Gamma_{i j}$ as before

$$
\Gamma_{i j}= \begin{cases}\frac{\lambda_{x}^{2} \Delta t_{u}}{\lambda^{2}(\Delta x)^{2}} & \text { if } \Delta \vec{x}_{i j}= \pm \Delta_{x} \hat{e}_{x} \\ \frac{\lambda_{z}^{2} \Delta t_{u}}{\lambda^{2}(\Delta z)^{2}} & \text { if } \Delta \vec{x}_{i j}= \pm \Delta_{z} \hat{e}_{z} \\ 0 & \text { if } \vec{x}_{i}=\vec{x}_{j}\end{cases}
$$

where $\Delta t_{u}$ is the time step of the jump process. The loss term $-u$ removes the diffusing agent with probability $\Delta t_{u}$ 
from the lattice. So the jump probabilities do not sum to one but obey $\sum_{i \in \mathrm{NN}(j)} \Gamma_{i j}=1-\Delta t_{u}$. This relation also fixes the time step $\Delta t_{u i}$. Finally, the source term $u_{0}$ defined in eq. (14) adds $\Delta t_{u i} u_{0 i}$ per time step. In total, we introduce the time-dependent lattice process for the control force variable $u_{i}$,

$$
u_{i}^{(n+1)}=\sum_{j} \Gamma_{i j} u_{j}^{(n)}+\Delta t_{u i} u_{0 i}
$$

which in steady state is equivalent to solving eq. (7) and thereby determines the optimal control force $u_{i}$. Since discretizing the source term $\Delta t_{u i} u_{0 i}$ requires special care, we explain it in the appendix B.

To solve eqs. (18), (20), and (22), we use an iterative scheme. For given $u^{\alpha}$, we determine the steady-state solutions $W^{(\alpha)}, V^{(\alpha)}$ of eqs. (18), (20), which then should give a refined control force $u^{\alpha+1}$ from the steady-state solution of eq. (22) using $W^{(\alpha)}, V^{(\alpha)}$. Then the iteration step starts anew. Typically, we start the iteration with $u^{(0)}(\vec{x})=0$. However, the scheme just outlined produces oscillations in $u$ in the case of particle sorting since it tries to alternately optimize the trajectories of the different particle types. To prevent these oscillations, we introduce a relaxation dynamics for the control force $u$ also used, for example, in training neural networks [32],

$$
u^{(\alpha+1)}=\left(1-\gamma^{(\alpha)}\right) u^{(\alpha)}+\gamma^{(\alpha)} \bar{u}^{(\alpha+1)}\left(W^{(\alpha)}, V^{(\alpha)}\right) .
$$

Here the relaxation or update rate $\gamma^{(\alpha)}$ assumes small and $\alpha$-dependent values so that $u^{(\alpha+1)}$ relaxes towards the steady-state solution $\bar{u}^{\alpha+1}\left(W^{(\alpha)}, V^{(\alpha)}\right)$ of eq. (22). We chose the update rate as $\gamma^{(\alpha)}=0.1 /(1+\beta \times \alpha)$, where $\beta=0.02$ for the single particle steering and $\beta=0.05$ for particle separation. With this procedure $u(\vec{x})$ indeed relaxed towards a steady-state solution of eq. (22), where we checked that always $\gamma^{(\alpha)} \geq 10^{-4}$.

\section{Application to inertial microfluidics}

Now we explain how we realize optimal control of particle steering and separation within inertial microfluidics.

\subsection{The system}

We study a rectangular channel with fixed width $2 w$, height $2 h$, and varying length $L$ as illustrated in fig. 2 . The channel axis points along the $z$-direction and $x=y=0$ corresponds to the center of the channel cross-section. We concentrate here on a channel aspect ratio $w / h \approx 0.42$. Since it is sufficiently smaller than one, we can restrict the particle dynamics to the $y=0$ plane as explained in sect. 3.2. A particle position in this plane is denoted by $\vec{x}=(x, z)$. The channel is filled by a Newtonian fluid with density $\rho$ and kinematic viscosity $\nu$ which exhibits a pressure-driven Hagen-Poiseuille flow profile [33]. The maximum flow velocity $v_{0}$ in the channel center determines the Reynolds number $\operatorname{Re}=2 w v_{0} / \nu$ [34]. The
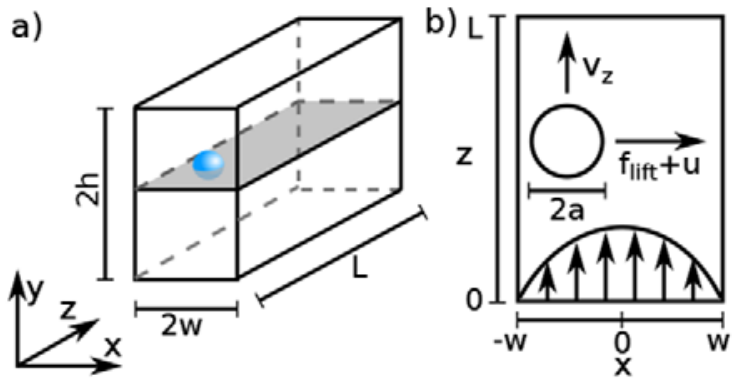

Fig. 2. a) Schematic of the rectangular channel with width $2 w$, height $2 h$ and length $L$. The two-dimensional plane relevant for the colloidal dynamics is shown in grey. b) The $(x, z)$ plane where the dynamics of the colloid takes place. The pressuredriven flow has a parabolic profile. The colloid of radius $a$ moves with velocity $v_{z}(x)$ along the channel in the $z$-direction. In the lateral $x$-direction it experiences the internal lift force $f_{\text {lift }}(x)$ and the external control force $u(x, z)$.

fluid contains a spherical particle with radius $a$ and mass $M=4 / 3 \pi a^{3} \rho$. Here, we fix the Reynolds number to $\operatorname{Re}=10$ and use two different particle sizes $a / w=0.3$ and $a / w=0.4$, which are typical experimental values $[7,8,18]$.

We determine the flow field within the channel and inertial lift forces acting on suspended particles using a mesoscopic simulation technique called Multi-Particle Collision Dynamics (MPCD) which effectively solves the Navier-Stokes equations [35-37]. We have recently used it to study the motion of sheets and active particles as well as the locomotion of the parasite African trypanosome [38-41]. Details of the MPCD technique applied to inertial microfluidics have been described in [25]. In contrast to our previous investigations we use here a channel with rectangular cross-section.

\subsection{Lift forces}

Since fluid inertia cannot be neglected at finite Reynolds numbers, the embedded particle experiences a lift force in the lateral channel direction [17]. The lift force has two contributions: a wall-induced component directed towards the channel center and a component induced by the nonuniform shear gradient of the Poiseuille flow which is directed towards the bounding walls [19].

Using MPCD, we determined both components of the lift force in the $x, y$ plane, $f_{\text {lift }, x}$ and $f_{\text {lift }, y}$, and also the axial velocity $v_{z}$ of the colloid. Close to the centerline at $x=y=0$, where the influence of the walls can be neglected, we find that the lift force scales with the particle size $a / w$ and Reynolds number Re as

$$
f_{\text {lift }} \propto\left(\frac{a}{w}\right)^{m} \operatorname{Re}^{2}
$$

The exponent $m \approx 3$ agrees with our earlier findings on circular channels [25] and results for rectangular channels [18]. 


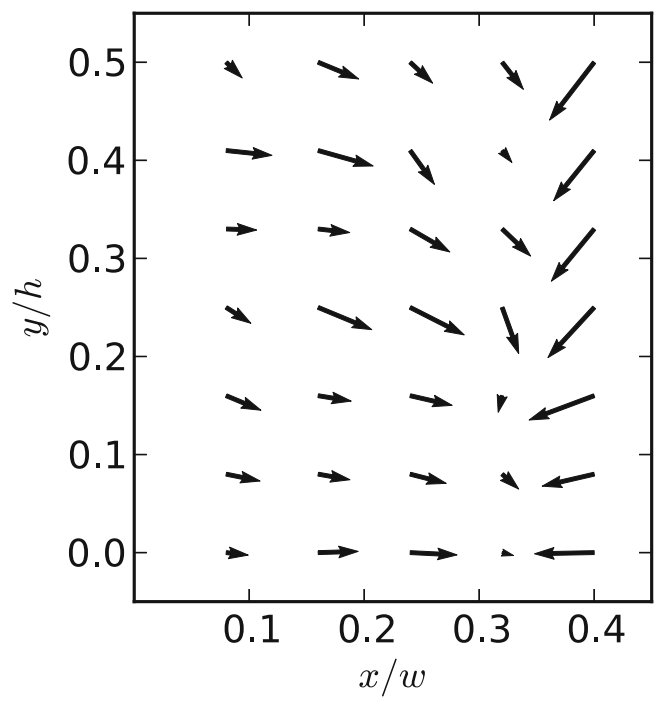

Fig. 3. The profile of the lift force $\left(f_{\text {lift }, x}, f_{\text {lift }, y}\right)$ in the crosssectional $x, y$ plane for particle size $a / w=0.4$ and Reynolds number $\operatorname{Re}=10$. Note the different scales in $x$ - and $y$ direction. Since the height of the channel is larger than its width $(w / h=0.42)$, the particle always experiences a force towards the central plane at $y=0$.

We already mentioned in the introduction that in channels with sufficiently large aspect ratio the lift forces along the longer cross-sectional axis always point towards the center plane of the channel $(y=0$ in our case, see fig. 2). This leaves only two stable positions along the $x$-axis each halfway between channel center and the walls [18]. In particular, the fact that the particles always experience a force which pushes them towards the center plane constrains the colloidal dynamics to two dimensions. This has already been used in experiments for sorting cells $[13,21]$. Now, fig. 3 shows an example for the crosssectional lift-force profile for particle radius $a / w=0.4$. Indeed, the lift-force component along the $y$-direction is always negative in the upper half of the channel and directed towards the center plane at $y=0$. The profile for $a / w=0.3$ (not shown) looks qualitatively the same. Hence, in the following we will concentrate on the particle dynamics in the $y=0$ plane.

In fig. 4 we plot the lift force and axial particle velocity at $y=0$ versus the $x$ coordinate. The results of the MPCD simulations (symbols) are fitted to appropriate functions with a least-squares fit (solid lines). Details of the fit are described in appendix C. We will use the analytic expressions of the fits when determining the optimal control force for particle steering and separation. Note that $f_{\text {lift }}=0$ indicates steady-state positions of the particles. While the position at $x=0$ is unstable, we have two stable locations which we denote by $\pm\left|x_{\text {eq }}\right|$.

\subsection{Langevin dynamics of the particle}

Using the inertial lift force $f_{\text {lift }}(x)=f_{\text {lift }, x}(x, y=0)$, the axial velocity $v_{z}(x)$, and an appropriate control force a)

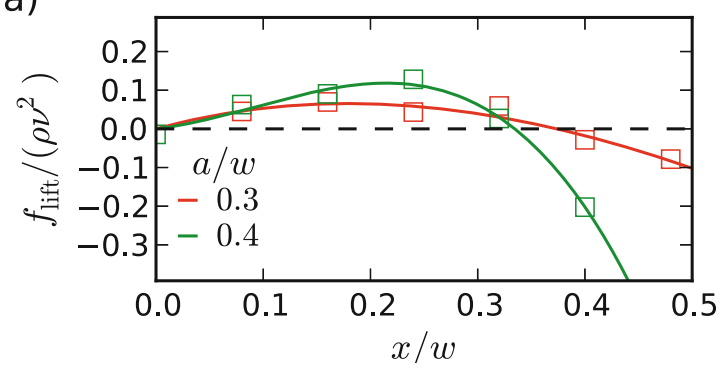

b)

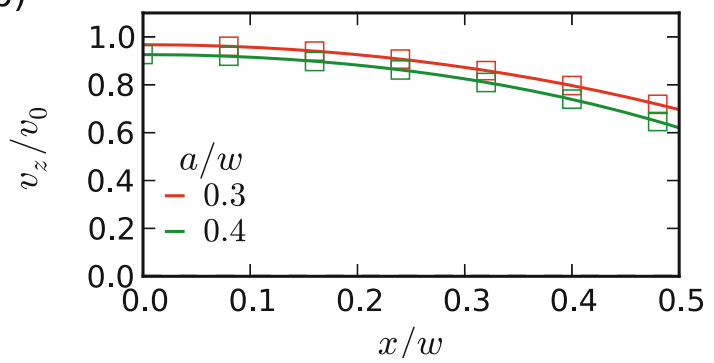

Fig. 4. The inertial lift forces (a) and axial particle velocities (b) at $y=0$ plotted versus the lateral position $x$ for $a / w=0.3$ (red) and $a / w=0.4$ (green). The symbols are obtained from MPCD simulations and the lines for the lift forces are fits to eq. (C.1) together with (C.2) while for $v_{z}(x)$ a parabolic fit to $c_{0}-c_{2} x^{2}-c_{4} x^{4}$ is used and $v_{0}$ is the maximum flow velocity of the applied Poiseuille flow.

$u(x, z)$, we can formulate the overdamped Langevin dynamics of a single particle in the $y=0$ plane of the microfluidic channel. It is equivalent to the description using the Smoluchowski equation (1) together with the operator $(4)$

$$
\begin{aligned}
\frac{\mathrm{d}}{\mathrm{d} t} x & =\xi^{-1}\left[f_{\text {lift }}(x)+u(x, z)\right]+\eta(t), \\
\frac{\mathrm{d}}{\mathrm{d} t} z & =v_{z}(x)
\end{aligned}
$$

Here, $\xi$ is the Stokes friction coeffcient of the particle. The stochastic force $\eta(t)$ is of thermal origin, has a zero mean, $\langle\eta(t)\rangle=0$, and its second moment obeys the fluctuationdissipation theorem: $\left\langle\eta(t) \eta\left(t^{\prime}\right)\right\rangle=2 D \delta\left(t-t^{\prime}\right)$ with diffusion constant $D=k_{B} T / \xi$. We now discuss several aspects of these equations.

To realize the spatially varying control force $u(\vec{x})$ acting on the particle inside the channel, one can think of optical tweezers. They can produce non-uniform force profiles as demonstrated, for example, with scanning tweezers in refs. [42-44] or holographic optical tweezers in refs. [45-47]. For simplicity, we assume here that the strength of the control force does not depend on the particle size. This approximation is valid for particles large compared to the wavelength of light [48]. In the following, we will choose the maximum control force $u_{\max }$ always larger than the local maxium of the lift force for $a / w=0.4$ but of the same order of magnitude. So we take $u_{\max }=0.264 \rho \nu^{2}=264 \mathrm{pN}$, which is accessible in experiments with optical tweezers [49-51]. 
Typically, the axial velocity $v_{z}(x)$ is much larger than the lateral drift velocities $\xi^{-1} f_{\text {lift }}$ and $\xi^{-1} u_{\max }$ (ca. by a factor of $\left.10^{2}-10^{3}\right)$. In the dynamics of the $z$ coordinate in eq. (26), we therefore neglect any effect the realization of the control force might have on the axial particle motion. Furthermore, we also neglect thermal motion in axial direction. Finally, we assume very dilute particle suspensions so that we do not have to consider any interactions between the particles.

In steady state the lateral $x$-position of the particle is distributed according to the Boltzmann distribution

$$
\rho_{0}(x) \propto \exp \left[\frac{1}{k_{B} T} \int_{0}^{x} \mathrm{~d} x^{\prime} f_{\text {lift }}\left(x^{\prime}\right)\right] .
$$

In ref. [25] we have demonstrated that this probability density agrees with the particle distributions observed in full hydrodynamic simulations. Since typically $f_{\text {lift }} a \gg$ $k_{B} T$, the Boltzmann distribution is strongly peaked about the steady-state positions on the positive and negative $x$ axis. So thermal motion hardly broadens the two peaks.

\section{Optimal control in inertial microfluidics}

In this section we present our results on how optimal control theory helps to steer single particles and to separate differently sized particles in a microfluidic channel with rectangular cross-section. In the following we always assume that the particles had sufficient time to settle into the central $y=0$ plane. Here they order along the lateral $x$-direction according to the Boltzmann distribution $\rho_{0}$ of eq. (27) about the steady-state positions $x_{\text {eq }}$ in both halves of the channel. This is the situation from which we start at our channel inlet.

\subsection{Steering single particles}

We illustrate the basic mechanism of the optimal control as introduced in sect. 2 by steering a single particle to a defined target. As an example we consider a particle with radius $a / w=0.4$ in a channel of length $L=200 w$ using a maximum allowed control force $u_{\max }=0.264 \rho \nu^{2}$. Without control the two equilibrium positions are $x_{\text {eq }} \approx \pm 0.33 w$ about which the initial distribution is strongly peaked. By applying the control force, we can direct the particles into the desired target interval. For the target interval $[0.05 w, 0.15 w]$, we illustrate the necessary control force in fig. 5 by color-coding it in the channel plane. To verify that these control forces are indeed able to steer particles, we simulated about 100 particle trajectories using the Langevin equations (25) and (26). They are shown as black lines.

We observe that the control force is mostly zero $\left(u / u_{\max }<10^{-14}\right)$ except in two narrow regions close to the trajectories. This feature does not change qualitatively when we vary the control-cost parameter $\lambda$ introduced in eq. (6). Decreasing it from $\lambda^{2}=10^{-3}$ to $10^{-5}$, the regions

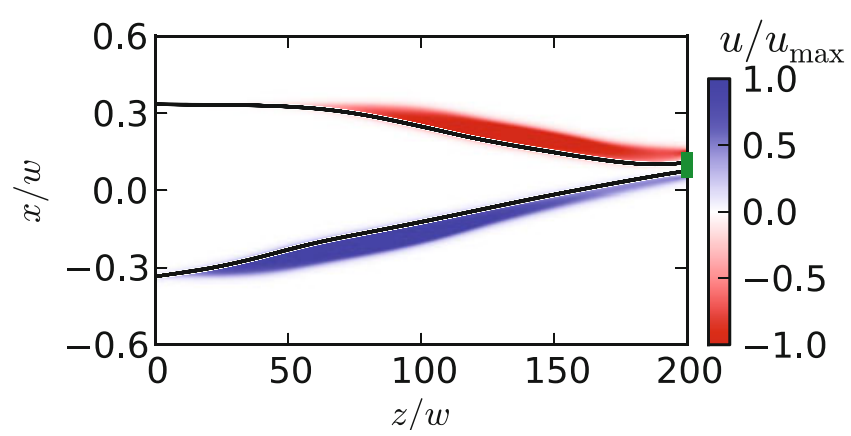

Fig. 5. The color-coded control force in the $x, z$ plane necessary to steer a particle with $a / w=0.4$ from its equilibrium positions at $\pm 0.33 w$ into the target interval $[0.05 w, 0.15 w]$ (green bar at $z / w=200$ ). The maximum control force is given by $u_{\max }=0.264 \rho \nu^{2}$. The black lines show 100 trajectories determined by simulating the Langevin equations (25) and (26). The regularization parameters are given by $\lambda^{2}=10^{-3}, \lambda_{x}^{2}=10^{-7}$, and $\lambda_{z}^{2}=10^{-4}$.

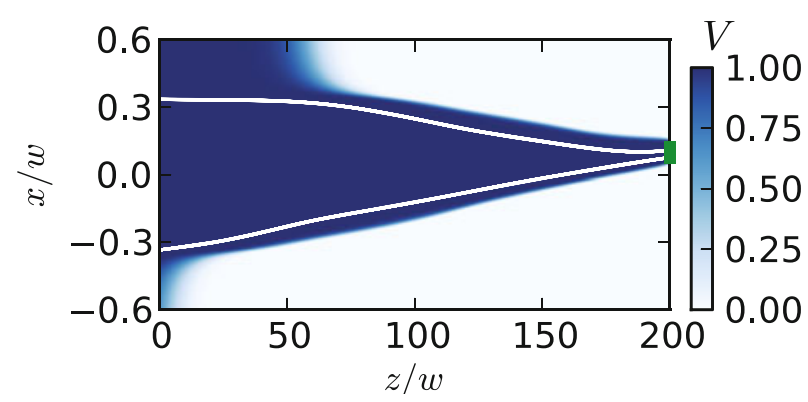

Fig. 6. The value function $V(x, z)$ for the situation shown in fig. 5. The white lines show the particle trajectories. The control force illustrated in fig. 5 significantly deviates from zero at large gradients of the value function as predicted by eqs. (7) and (8).

of non-zero control force are just more extended since nonzero control "costs less", whereas for $\lambda^{2}=10^{-1}$ the regions are more concentrated. So $\lambda$ can be used to design the action of the control force according to the experimental needs.

According to eqs. (7) and (8), we expect the control force to be concentrated in regions where the value function $V$ has large gradients meaning that the control forces push the system to larger probabilities to reach the target. This is confirmed by fig. 6 , where we show the value function in blue: dark colors mean higher probability to reach the target. The control force in fig. 5 follows very nicely the sharp edge of the value function.

By changing the position of the target and its width, we are able to steer the particle towards almost all available positions at the channel outlet. It is just difficult to place the particles close to the walls due to the strong repulsion from the walls. In fig. 7 we plot the peaked particle distributions at the channel outlet for a target position $x_{t} / w=0.2$ and different target widths $w_{t}$. The width of the peaked distributions is independent of the target width and solely determined by temperature. Since we 


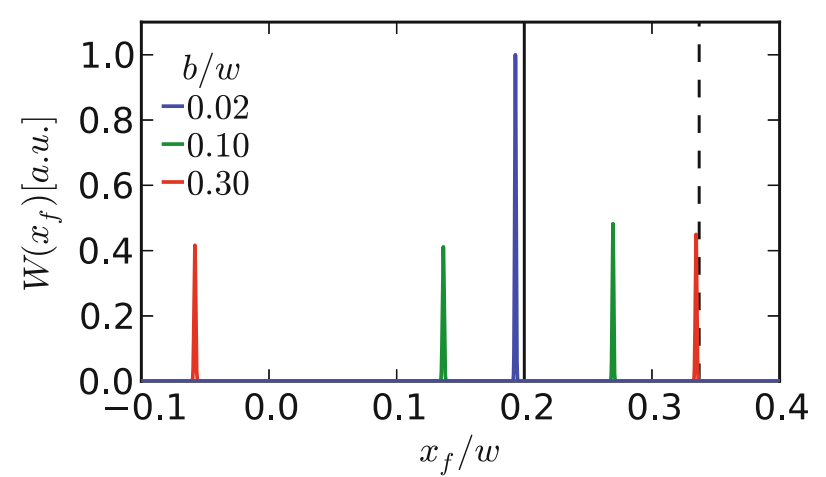

Fig. 7. The distribution $W\left(x_{f}\right)$ of the particle position at the channel outlet for different target widths $b$. The target position is $x_{t} / w=0.2$ (vertical black line) and temperature is $T=300 \mathrm{~K}$. The widths of the individual peaks are mainly determined by the temperature and does not show any pronounced dependence on other parameters. For $b / w=0.3$, one half of the particles stay at their equilibrium position at $0.33 \mathrm{w}$ (black dashed line).

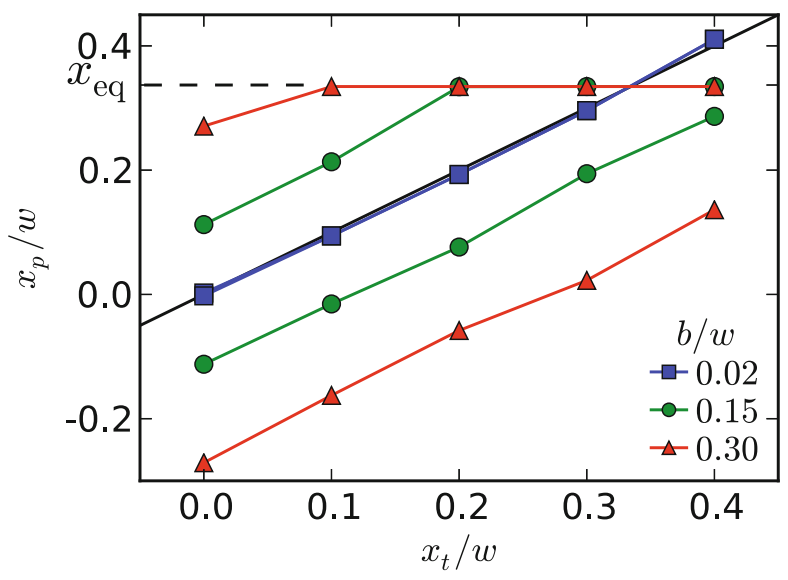

Fig. 8. The position $x_{p}$ of the particles at the outlet plotted versus target position $x_{t}$ for different target widths $2 b$. The line $x_{p}=x_{t}$ is shown in black and the equilibrium position $x_{\text {eq }}$ of the particles for zero control force is indicated.

only optimize the probability of the particle to reach the target, its exact position at the outlet does not influence the outcome. As a result we generally find two positions at the channel outlet corresponding to the two equilibrium positions at the channel inlet. Only for the smallest target widths do both positions coincide since the two particle trajectories starting at the inlet merge before reaching the outlet.

In fig. 8 we demonstrate that we can place particles at almost all positions $x_{p}$ at the outlet by tuning the target position $x_{t}$ and width $2 b$ appropriately. If the equilibrium position at $x_{\mathrm{eq}}=0.33$ resides inside the target interval, we typically find that the particles simply stay there (upper red and green lines in fig. 8). Only the particles from the second equilibrium position at $x_{\text {eq }}=-0.33$ need to be steered into the target. When the target width is sufficiently small (blue line in fig. 8), the two particle trajectories merge. They can even be placed between the equilibrium position $x_{\mathrm{eq}}=0.33$ and the wall, where the repulsive wall force becomes large.

\subsection{Separation of particles based on their size}

We now demonstrate how we can separate two types of particles of different size. We only use here their differences in the strength and profile of the lift forces. The strength scales as the third power of particle radius near the channel center $[18,25]$. As already mentioned in the introduction, Mach et al. [13] used this scaling to separate a stream of particles. Whereas the larger particles were focussed to specific positions in the channel, the lift forces acting on the smaller particles were small so that the particles kept their roughly uniform distribution in the channel cross-section. Since we use here an additional external control force, we are able to focus both particle types to specific locations which greatly enhances the efficiency of particle separation.

In our first example, we consider a stream with two types of particles with respective radii $a_{1} / w=0.3$ and $a_{2} / w=0.4$ which we try to steer towards $x_{t, 1} / w=0.3$ and $x_{t, 2} / w=-0.3$, respectively. The target width in both cases is $2 b=0.2$. The equilibrium positions of both the larger $\left(x_{\mathrm{eq}}= \pm 0.33 w\right)$ and the smaller $\left(x_{\mathrm{eq}}= \pm 0.38 w\right)$ particles are inside the target regions. However, since both particle types occur at their two equilibrium positions at the channel inlet, we have to move the particles across the channel center to separate them. This is demonstrated in fig. 9(a) for a channel of length $L / w=300$. We colorcode the necessary control force profile to separate the two particle types and show the resulting particle trajectories. Starting from its positive equilibrium position, the smaller particle is slightly pushed outwards and then relaxes to its equilibrium position at the channel outlet. Similarly, the larger particle at its negative equilibrium position is slightly moved towards the channel center and then also relaxes towards the equilibrium position with the help of a weak control force. Particles located in the "wrong" half of the channel are pulled together in the lower channel half. Then, the control force together with the two different lift force profiles separates both particle types and, finally, they are steered into their respective targets.

For the force profile in fig. 9(a), we illustrate the corresponding value function $V=V_{1}+V_{2}$ as a sum of the value functions for both particle types in fig. 10. As indicated, in the regions with $V \approx 1$ particles of either type 1 or 2 reach their targets with certainty when started in the respective region whereas the other particle type fails. Only in the red region with $V=2$ do both particle types reach their targets. The control force profile to achieve this behavior is concentrated around the gradients in $V$.

In fig. 9(b) we illustrate an even more challenging particle separation. We push the targets further apart and strongly reduce their widths so that they no longer overlap with the equilibrium positions of the particles which have to be pushed against the channel walls. However, even then we are able to separate both particle types. 

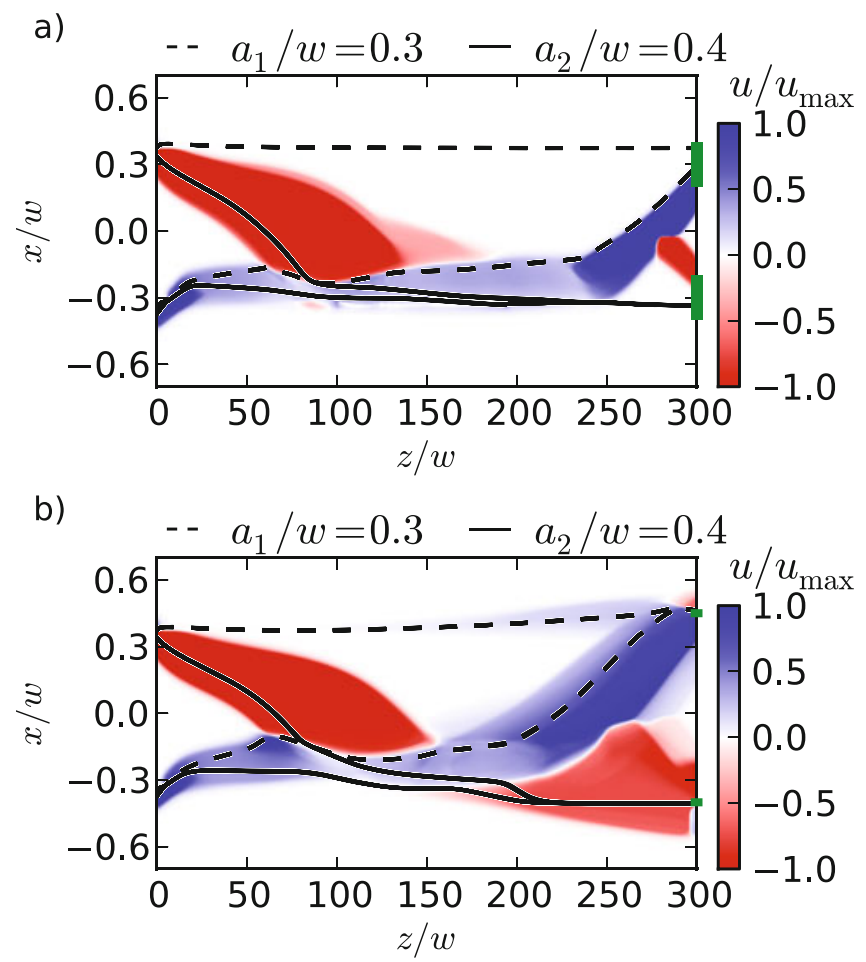

Fig. 9. a) The trajectories of two types of particles with radii $a_{1} / w=0.3$ (dashed line) and $a_{2} / w=0.4$ (solid line) that are steered under the action of the color-coded control force $u(x, z) / u_{\max }$ into their targets at the channel outlet (green bar). Maximum control force is $u_{\max }=0.264 \rho \nu^{2}$. The target positions are $x_{t, 1} / w=0.3, x_{t, 2} / w=-0.3$, and the widths are $2 b / w=0.2$. b) Same as in a) but now the target positions are $x_{t, 1} / w=0.45, x_{t, 2} / w=-0.4$ and the widths are $2 b / w=0.04$.

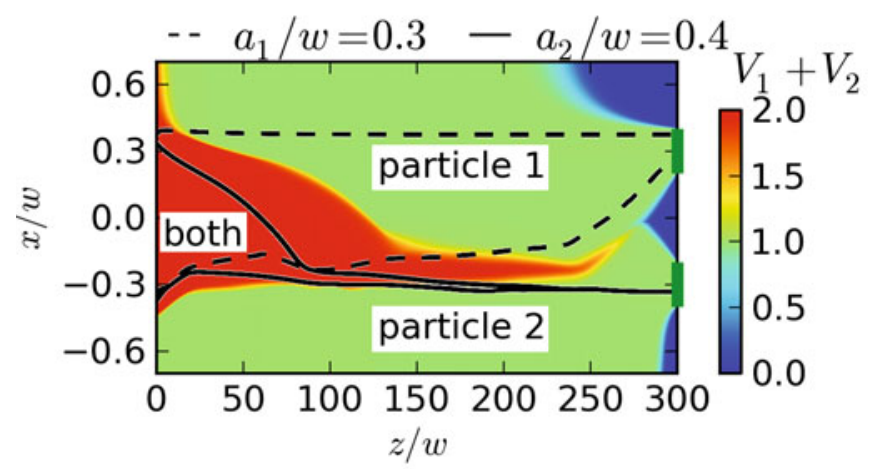

Fig. 10. Total value function $V=V_{1}(x, z)+V_{2}(x, z)$ for the system of fig. 9. Inside the green regions either particles of type 1 or 2 reach their targets with certainty whereas the other type fails.

In the third example we keep the particle sizes as before but place the target for the smaller particles into the center of the channel, while the larger particles are steered towards two targets near to their equilibrium positions. Figure 11(a) illustrates how this setup can be used to separate the two particle types. Behind the target line (green dashed line) a bifurcating channel with three outlets is placed. Whereas the large particles are advected a)
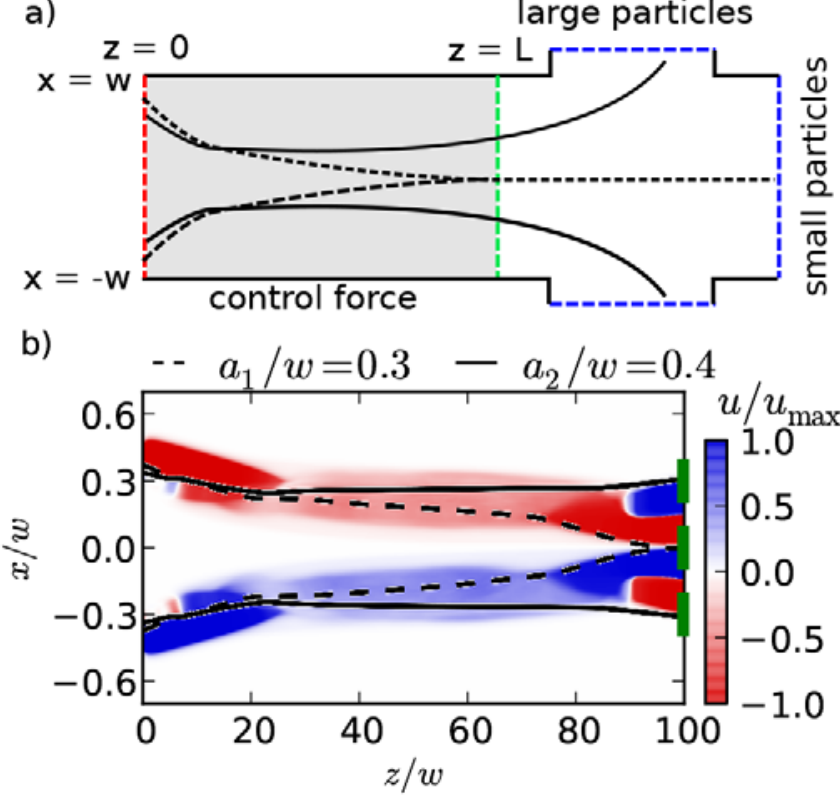

Fig. 11. (a) Schematic microfluidic setup for particle separation. Particles enter the system from the inlet (red dashed line) at their equilibrium positions and flow through the designed control force profile that steers particles to prescribed positions at $z=L$. A bifurcating channel as in ref. [13] then sorts the large particles into the side channels and the small particles into the center outlets. (b) The trajectories of two types of particles with radii $a_{1} / w=0.3$ (dashed line) and $a_{2} / w=0.4$ (solid line) initiated by the color-coded control force $u(x, z) / u_{\max }$. Small and large particles are steered into respective targets (green bars) at $x_{t, 1} / w=0$ and $x_{t, 2} / w=0.3$; the width is $2 b=0.2 w$.

to the side channels (solid lines), the small particles flow on a straight path (dashed line) and are collected in the third outlet. Exactly this type of bifurcating channel was used in the experiments of Mach et al. to separate bacteria from red blood cells [13]. However, whereas the large blood cells could be collected in the side channels, the smaller bacteria were still uniformly distributed. Their lift forces are smaller and need longer channels to focus them. As a result, some of the bacteria also entered the side channels and the separation was not perfect.

By using the opimized control force profile $u$ of fig. 11(b) we can focus the smaller particles from their two equilibrium positions to the channel center and thereby improve the efficiency of separation. A closer look at the particle trajectories shows that the equilibrium positions at $z=0$ of the larger particles (solid line) are slightly closer to the channel center compared to the smaller particle. So the particle trajectories have to be swapped by the control force to move the smaller particles to the center. This is indeed accomplished by the control force in combination with the lift forces.

In an experimental setting one always has noise and imperfections. This will presumably also apply to the control force. We therefore add spatial noise to the control force which then consists of the deterministic part and a 


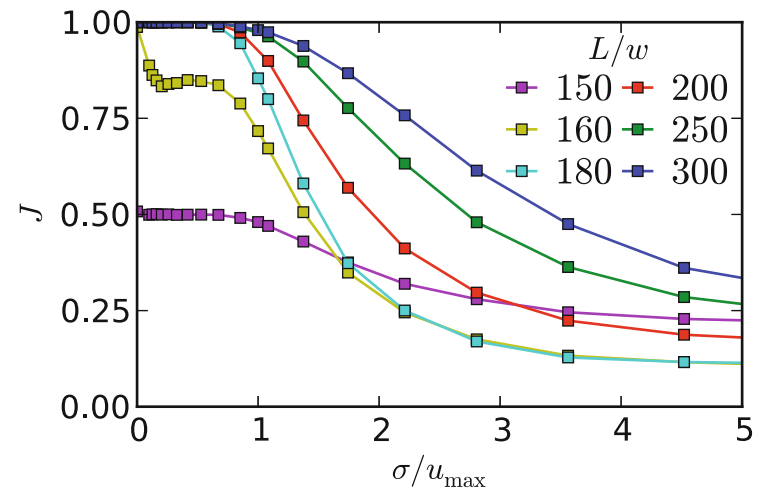

Fig. 12. Separation probability $J=J_{1} J_{2}$ as a function of noise amplitude of the control force, $\sigma / u_{\max }$, and different channel lengths $L / w$ for the set up of fig. 9. The maximum deterministic control force is $u_{\max }=0.226 \rho \nu^{2}$.

stochastic component $\eta$

$$
u_{\text {noise }}(x, z)=u(x, z)+\eta(x, z) .
$$

Here, the noise $\eta$ is delta correlated in space with zero mean and variance $\left\langle\eta(x, z) \eta\left(x^{\prime}, z^{\prime}\right)\right\rangle=\sigma^{2} \delta\left(x-x^{\prime}\right) \delta\left(z-z^{\prime}\right)$. For our first example, where we separate the small and large particles into the two halves of the channel (see fig. $9(\mathrm{a})$ ), we determined the separation probability $J=$ $J_{1} J_{2}$ versus reduced noise amplitude $\sigma / u_{\max }$ for different channel lengths. The results are plotted in fig. 12. Surprisingly, up to a noise strength of $\sigma / u_{\max }=1$ and for sufficiently long channels the separation is perfect $(J=1)$ meaning that our method is very robust against noise. This is also illustrated in fig. 13, where we plot the particle distributions of the two types at the outlet for two noise values $\sigma / u_{\max }=1$ and 2.212. So, increasing the noise beyond $\sigma / u_{\max }=1$ decreases the separation probability continuously. We also observe that perfect separation is only possible beyond a certain length $L$ of the channel. A sharp transition at $\sigma=0$ takes place between sorting both particle types $(L / w \geq 160)$ and steering only one type in its specific target $(\bar{L} / w=150)$ while only half of the particles of the other type reach their target due to their initial equilibrium position.

\section{Conclusion}

Particle steering and separation in microfluidic channels is essential, for example, for biomedical applications. Here we demonstrated how concepts from optimal control theory help to design optimal control force profiles. In the inertial regime they steer particles in pressuredriven Poiseuille flow. In particluar, for single particles we designed control forces that are concentrated on narrow regions within the microfluidic channel and thereby minimize the experimental efforts. These contol forces can guide a particle train already focussed by inertial lift forces
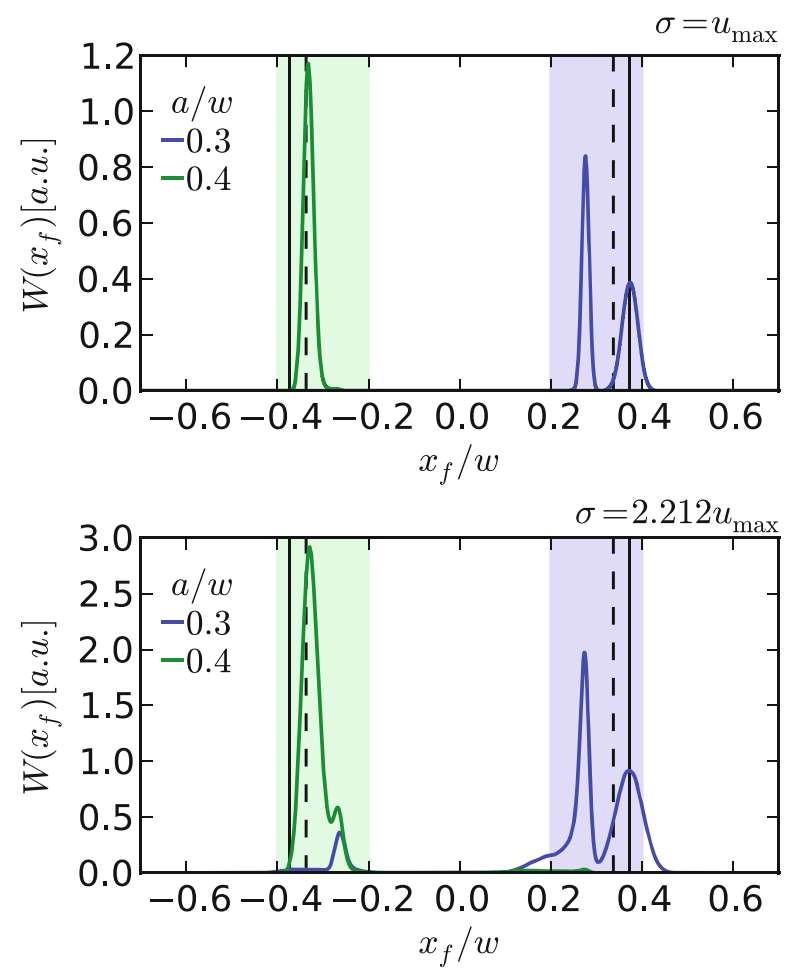

Fig. 13. Particle distributions of the two particle types at the channel outlet. At noise value $\sigma=u_{\max }$ the particle separation is still perfect, however even at $\sigma=2.212 u_{\max }$ all large particles (green) are sorted in the correct target whereas some of the small particles (blue) miss the right target. The targets are indicated by rectangles shaded in the color of the particle type. Solid and dashed black lines show the respective equilibrium positions of the small and large particles. The channel length is $L=300 \mathrm{w}$ and the maximum deterministic control force is $u_{\max }=0.226 \rho \nu^{2}$.

to almost any position at the channel outlet. We then applied our approach to separate particles of different types. Remarkably, we achieve this by one single control force profile which acts on both particle types and steers them to well-separated positions at the channel outlet. The separation of particles solely relies on the difference in the lift-force pofiles and thereby allows to separate particles of similar sizes. Our approach is even robust against strong stochastic variations in the control force which may occur in an experiment. This all goes well beyond existing experimental methods within inertial microfluidics [13] and therefore improves the possibility of particle separation and filtering in the inertial regime.

Scanning and holographic optical tweezers are a wellestablished method to create an optical landscape which acts with a force on colloidal objects [42-47]. The method is ideal for realizing the optimized control force profiles determined in this article. However, also other external fields such as electric or magnetic fields are employed for particle sorting and separation $[2,3]$. Combining these established approaches with an optimal design for tuning the strength of the applied fields would certainly enhance their capability for steering particles. 
Future work will study how external fields are used for optimal particle steering, in particular, within inertial microfluidics. External fields not only act directly on the particles. By slowing the particles down or enhancing their velocities along the channel axis, also the lift force profiles are modified $[52,53]$. Furthermore, so far we looked at dilute suspensions where hydrodynamic interactions between the particles are negligible. However, for highthroughput systems it is desirable to increase the number of particles flowing through the microfluidic device, so collective effects become important [54-56]. We will address them in future work and investigate how optimal control theory presented here has to be modified for steering interacting particles.

We thank P. Cicuta, F. Schulze, and K. Wolff for useful discussions. We also acknowledge support by the Deutsche Forschungsgemeinschaft in the framework of the collaborative research center SFB 910.

\section{Appendix A. Conditions for the optimal control force}

The objective functional $J^{\text {tot }}$ of eq. (6) has to be maximized under the constraint that the probability density of the particle, $\rho(\vec{x}, t)$, satisfies the Smoluchowski equation (1).

In eq. (5) we give the probability of the particle to reach the target at $z=L$. We first rewrite it as follows. We use $j_{z}(\vec{x}, t)=\rho(\vec{x}, t) v_{z}(x)$ and introduce the cumulated or time-integrated density $W(\vec{x})=\int_{0}^{\infty} \mathrm{d} t \rho(\vec{x}, t)$ to arrive at

$$
J=\int_{-w}^{w} \mathrm{~d} x v_{z}(x) W(x, L) R(x) .
$$

Integrating the Smoluchowski equation (1) over time, the cumulated density inside the channel satisfies $\mathcal{L}[u] W(\vec{x})=$ 0 . Contributions from the time integration at $t=0$ and $\infty$ vanish. Initially there is no particle inside the channel and at $t=\infty$ the particle has left the finite channel domain we consider. The boundary condition $j_{x}=0$ at the channel walls translates into

$$
j_{x}^{W}( \pm w, z)=0
$$

where we have defined $j_{x}^{W}(\vec{x})=\left(\xi^{-1}\left[f_{\text {lift }}(x)+u(\vec{x})\right]-\right.$ $\left.D \frac{\partial}{\partial x}\right) W(\vec{x})$, and at the channel inlet we have

$$
W(x, 0)=\rho_{0}(x) .
$$

Now, maximizing the objective functional $J^{\text {tot }}$ under the constraints for the cumulated density $W(\vec{x})$, one has to consider the following Lagrangian functional:

$$
\begin{aligned}
J_{C}^{\mathrm{tot}}= & \int_{-w}^{+w} \mathrm{~d} x v_{z}(x) W(x, L) R(x)-\frac{1}{2} \int_{\Omega} \mathrm{d}^{2} x\left[\lambda^{2}|u(x, z)|^{2}\right. \\
& \left.+\lambda_{x}^{2}\left(\frac{\partial u(x, z)}{\partial x}\right)^{2}+\lambda_{z}^{2}\left(\frac{\partial u(x, z)}{\partial z}\right)^{2}\right] \\
& +\int_{\Omega} \mathrm{d}^{2} x V(\vec{x}) \mathcal{L}[u] W(\vec{x}) \\
& +\int_{0}^{L} \mathrm{~d} z \phi_{ \pm w}(z) j_{x}^{W}( \pm w, z) \\
& +\int_{-w}^{+w} \mathrm{~d} x \phi_{i}(x)\left[W(x, 0)-\rho_{0}(x)\right]
\end{aligned}
$$

where we introduced the Lagrange multipliers $V$ (adjoint state), $\phi_{ \pm w}$, and $\phi_{i}$ to enforce, respectively, the stationary Smoluchowski equation for $W(\vec{x})$ as well as the boundary condition at the channel walls and the initial condition.

Variation of $J_{C}^{\text {tot }}$ with respect to $u(\vec{x})$ gives the already stated gradient equation for the optimal control force $u$,

$$
u(\vec{x})-\frac{\lambda_{x}^{2}}{\lambda^{2}} \frac{\partial^{2}}{\partial x^{2}} u(\vec{x})-\frac{\lambda_{z}^{2}}{\lambda^{2}} \frac{\partial^{2}}{\partial z^{2}} u(\vec{x})=u_{0}(\vec{x}),
$$

where

$$
u_{0}(\vec{x})=\frac{1}{\lambda^{2}} W(\vec{x}) \frac{\partial}{\partial u} \mathcal{L}^{+}[u] V(\vec{x})
$$

results from the first term in the second line of eq. (A.4). Note that $\mathcal{L}^{+}[u]$, in contrast to $\mathcal{L}[u]$, only depends on $u$ and not its derivative $\partial u / \partial x$.

Variation of $J_{C}^{\text {tot }}$ with respect to $V(\vec{x}), \phi_{ \pm w}$, and $\phi_{i}$ reproduces the stationary Smoluchowski equation for $W(\vec{x})$ as well as its boundary and initial conditions. On the other hand, variation with respect to $W(\vec{x})$ leads to the stationary Kolmogorov-Backward or adjoint equation for the value function, $\mathcal{L}^{+}[u] V(\vec{x})=0$, as well as the boundary condition $\frac{\partial}{\partial x} V(\vec{x})=0$ at the channel walls and $V(x, L)=R(x)$ at the channel outlet.

\section{Appendix B. Source term of the control force equation}

To solve eq. (7) for the optimal control force $u(\vec{x})$ on a lattice as implemented in eq. (22), we also have to discretize the source term $u_{0}(\vec{x})$ in eq. (14). In particular, we have to discretize the derivative of the value function $V_{i}(\vec{x})$ of particle type $i$. Typically, this is done using central differences. However, we made the experience that our control force $u$ oscillates during iteration and does converge slowly. This is due to situations as illustrated in fig. 14. Whereas the derivative of the value function $V_{1}$ is well represented by central differences, they do not reflect the shape of $V_{2}$. We, therefore, use forward and backward 


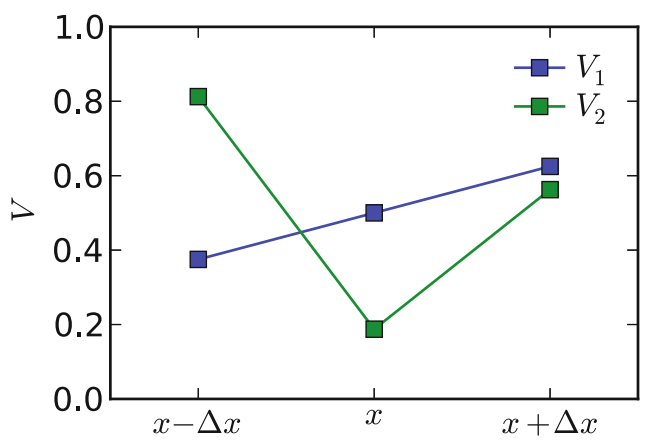

Fig. 14. Derivatives of the value functions. While for $V_{1}$ both central and one-sided differences agree, they give different values for $V_{2}$.

differences to disctretize the source term,

$$
u_{0}^{ \pm}(\vec{x})=\frac{1}{\lambda^{2}} \sum_{i} \frac{J}{J_{i} \xi_{i}} W_{i}(\vec{x}) \frac{ \pm\left[V_{i}(x \pm \Delta x, z)-V_{i}(x, z)\right]}{\Delta x},
$$

and use for $u_{0}$ the discretized value with the larger magnitude $\left|u_{0}^{+}\right|$or $\left|u_{0}^{-}\right|$.

\section{Appendix C. Fitting lift forces and axial velocities}

To use the lift forces obtained with MPCD for the optimal control of particle steering, we perform a least square fit to an ansatz function. It consists of a third-order polynomial to describe the shear-gradient contribution and a term which takes into account the divergence of the lift force close to the wall

$$
f_{\mathrm{cl}}(x)=\phi_{1} x+\phi_{3} x^{3}+\phi_{w} f_{\mathrm{wall}}(x),
$$

where we use

$$
f_{\mathrm{wall}}(x)=\frac{1}{x-(1+\delta) x_{\max }}+\frac{1}{x+(1+\delta) x_{\max }},
$$

$x_{\max }=w-a$ indicates the position of the particle when touching the wall at $x>0$. We model the divergence at the wall by $1 / x$ but avoid the singularity through the offset $\delta=10^{-3}$ which facilitates the fitting procedure.

Second, we fit the axial velocity by a fourth-order polynomial in the lateral coordinate $x$

$$
v_{z}(x)=\sum_{n=0}^{2} v_{n} x^{2 n}
$$

Open Access This is an open access article distributed under the terms of the Creative Commons Attribution License (http://creativecommons.org/licenses/by/3.0), which permits unrestricted use, distribution, and reproduction in any medium, provided the original work is properly cited.

\section{References}

1. M. Toner, D. Irimia, Annu. Rev. Biomed. Eng. 7, 77 (2005).

2. N. Pamme, Lab Chip 7, 1644 (2007).

3. H. Tsutsui, C.-M. Ho, Mech. Res. Commun. 36, 92 (2009).

4. T.M. Squires, S.R. Quake, Rev. Mod. Phys. 77, 977 (2005).

5. F.P. Bretherton, J. Fluid Mech. 14, 284 (1962).

6. A.A.S. Bhagat, S.S. Kuntaegowdanahalli, I. Papautsky, Microfluidics Nanofluidics 7, 217 (2009).

7. A.A.S. Bhagat, S.S. Kuntaegowdanahalli, I. Papautsky, Phys. Fluids 20, 101702 (2008).

8. S.C. Hur, H.T.K. Tse, D. Di Carlo, Lab Chip 10, 274 (2010).

9. S.S. Kuntaegowdanahalli, A.A.S. Bhagat, G. Kumar, I. Papautsky, Lab Chip 9, 2973 (2009).

10. J. Seo, M.H. Lean, A. Kole, Appl. Phys. Lett. 91, 033901 (2007).

11. D. Di Carlo, J.F. Edd, D. Irimia, R.G. Tompkins, M. Toner, Analyt. Chem. 80, 2204 (2008).

12. Z. Wu, B. Willing, J. Bjerketorp, J.K. Jansson, K. Hjort, Lab Chip 9, 1193 (2009).

13. A.J. Mach, D. Di Carlo, Biotechnol. Bioeng. 107, 302 (2010).

14. F. Tröltzsch, Optimal Control of Partial Differential Equations: Theory, Methods, and Applications, Graduate Studies in Mathematics (American Mathematical Society, 2010).

15. S.S. Sritharan, Optimal Control of Viscous Flow, Miscellaneous Titles in Applied Mathematics Series (Society for Industrial and Applied Mathematics, 1998).

16. M. Soner, Stochastic Optimal Control in Finance, Cattedra Galileiana (Edizioni della Normale, 2005).

17. G. Segré, A. Silberberg, Nature 189, 209 (1961).

18. D. Di Carlo, J.F. Edd, K.J. Humphry, H.A. Stone, M. Toner, Phys. Rev. Lett. 102, 094503 (2009).

19. J.P. Matas, J.F. Morris, E. Guazzelli, Oil Gas Sci. Technol. 59, 59 (2004).

20. J. Zhou, P.V. Giridhar, S. Kasper, I. Papautsky, Lab Chip 13, 1919 (2013).

21. S.C. Hur, A.J. Mach, D. Di Carlo, Biomicrofluidics 5, 022206 (2011).

22. E.S. Asmolov, J. Fluid Mech. 381, 63 (1999).

23. B.P. Ho, L.G. Leal, J. Fluid Mech. 65, 365 (1974).

24. B. Chun, A.J.C. Ladd, Phys. Fluids 18, 031704 (2006).

25. C. Prohm, M. Gierlak, H. Stark, Eur. Phys. J. E 35, 1 (2012).

26. R. Merton, Continuous-Time Finance, Macroeconomics and Finance Series (Wiley, 1992).

27. R.C. Merton, Rev. Econ. Statistics 51, 247 (1969).

28. R. Sutton, A. Barto, Reinforcement Learning: An Introduction, A Bradford book (MIT Press, 1998).

29. T. Schmiedl, U. Seifert, Phys. Rev. Lett. 98, 108301 (2007).

30. H.J. Kushner, P. Dupuis, Numerical Methods for Stochastic Control Problems in Continuous Time, Applications of Mathematics, Vol. 24 (Springer, 2001).

31. M. Boué, P. Dupuis, SIAM J. Numer. Anal. 36, 667 (1999).

32. Y. LeCun, L. Bottou, G. Orr, K. Muller, in Neural Networks: Tricks of the Trade, edited by G. Montavon, G.B. Orr, K.R. Müller (Springer, 1998).

33. H. Bruus, Theoretical Microfluidics (Oxford University Press, 2007). 
34. G. Batchelor, An Introduction to Fluid Dynamics (Cambridge University Press, 2000).

35. A. Malevanets, R. Kapral, J. Chem. Phys. 110, 8605 (1999).

36. A. Malevanets, R. Kapral, J. Chem. Phys. 112, 7260 (2000).

37. G. Gompper, T. Ihle, D. Kroll, R. Winkler, in Advanced Computer Simulation Approaches for Soft Matter Sciences III, Advances in Polymer Science (Springer Berlin Heidelberg, 2008) pp. 1-87.

38. S.B. Babu, H. Stark, Eur. Phys. J. E 34, 1 (2011).

39. A. Zöttl, H. Stark, Phys. Rev. Lett. 108, 218104 (2012).

40. M.T. Downton, H. Stark, J. Phys.: Condens. Matter 21 204101 (2009).

41. S.B. Babu, H. Stark, New J. Phys. 14, 085012 (2012).

42. D. Babič, C. Bechinger, Phys. Rev. Lett. 94, 148303 (2005).

43. S. Bleil, P. Reimann, C. Bechinger, Phys. Rev. E 75 031117 (2007).
44. N. Bruot, J. Kotar, F. de Lillo, M.C. Lagomarsino, P. Cicuta, Phys. Rev. Lett. 109, 164103 (2012).

45. D.G. Grier, Nature 424, 810 (2003).

46. K. Ladavac, K. Kasza, D.G. Grier, Phys. Rev. E 70, 010901 (2004).

47. M. Padgett, R. Di Leonardo, Lab Chip 11, 1196 (2011).

48. A. Ashkin, Biophys. J. 61, 569 (1992).

49. A. Jannasch, A.F. Demirörs, P.D. van Oostrum, A. van Blaaderen, E. Schäffer, Nat. Photonics 6, 469 (2012).

50. J. Dong, C.E. Castro, M.C. Boyce, M.J. Lang, S. Lindquist, Nat. Struct. Mol. Biol. 17, 1422 (2010).

51. B. Maier, L. Potter, M. So, H.S. Seifert, M.P. Sheetz, Proc. Natl. Acad. Sci. U.S.A. 99, 16012 (2002).

52. W.Y. Kim, J.Y. Yoo, Lab Chip 9, 1043 (2009).

53. C. Prohm, H. Stark, unpublished results.

54. W. Lee, H. Amini, H.A. Stone, D. Di Carlo, Proc. Natl. Acad. Sci. U.S.A. 107, 22413 (2010).

55. J.-P. Matas, V. Glezer, E. Guazzelli, J.F. Morris, Phys. Fluids 16, 4192 (2004).

56. D. Di Carlo, D. Irimia, R.G. Tompkins, M. Toner, Proc. Natl. Acad. Sci. U.S.A. 104, 18892 (2007). 